\title{
Nucleon structure in terms of OPE with non-perturbative Wilson coefficients
}

\author{
W. Bietenholz*a,b, N. Cundy ${ }^{\mathrm{b}}$, M. Göckeler ${ }^{\mathrm{b}}$, R. Horsley ${ }^{\mathrm{c}}$, \\ H. Perlt ${ }^{\mathrm{d}}$, D. Pleiter ${ }^{\mathrm{a}}$, P.E.L. Rakow ${ }^{\mathrm{e}}$, G. Schierholz ${ }^{\mathrm{a}}$, \\ A. Schiller ${ }^{\mathrm{d}}$ and J.M. Zanotti ${ }^{\mathrm{c}}$ \\ a John von Neumann Institut für Computing NIC, \\ Deutsches Elektron-Synchrotron DESY, 15738 Zeuthen, Germany \\ ${ }^{\mathrm{b}}$ Institut für Theoretische Physik, Universität Regensburg, 93040 Regensburg, Germany \\ c School of Physics, University of Edinburgh, Edinburgh EH9 3JZ, United Kingdom \\ ${ }^{\mathrm{d}}$ Institut für Theoretische Physik, Universität Leipzig, 04109 Leipzig, Germany \\ e Theoretical Physics Division, Dept. of Mathematical Sciences, University of Liverpool, \\ Liverpool, L69 3BX, United Kingdom
}

E-mail: bietenho@ifh.de

\begin{abstract}
Lattice calculations could boost our understanding of Deep Inelastic Scattering by evaluating moments of the Nucleon Structure Functions. To this end we study the product of electromagnetic currents between quark states. The Operator Product Expansion (OPE) decomposes it into matrix elements of local operators (depending on the quark momenta) and Wilson coefficients (as functions of the larger photon momenta). For consistency with the matrix elements, we evaluate a set of Wilson coefficients non-perturbatively, based on propagators for numerous momentum sources, on a $24^{3} \times 48$ lattice. The use of overlap quarks suppresses unwanted operator mixing and lattice artifacts. Results for the leading Wilson coefficients are extracted by means of Singular Value Decomposition.
\end{abstract}

The XXVI International Symposium on Lattice Field Theory

July 14 - 19, 2008

Williamsburg, Virginia, USA

\footnotetext{
${ }^{*}$ Speaker.
} 


\section{Motivation}

The computation of moments of the nucleon structure functions is a fascinating challenge: it is known to be diffi cult, but it is a point where lattice results could contribute much to the understanding and interpretation of phenomenological data from Deep Inelastic Scattering.

The continuum formulation of hadron structure functions is plagued by renormalon ambiguities, i.e. power-like IR contributions, see e.g. Refs. [1]. Here we refer to the lattice regularisation, where a general moment of the nucleon structure function can be expanded as

$$
\mathscr{M}\left(q^{2}\right)=c^{(2)}(a q) A_{2}(a)+c^{(4)}(a q) \frac{1}{q^{2}} A_{4}(a)+\ldots\{\text { higher twists }\} .
$$

It depends on the transfer momentum $q$, while $a$ is the lattice spacing, $c^{(2)}, c^{(4)} \ldots$ are Wilson coeffi cients (where the superscript is the twist), and $A_{2}, A_{4} \ldots$ are reduced matrix elements ("reduced" in the sense that the Lorentz structure is factored out).

For the evaluation of $A_{2}$ there is an established procedure, which employs the ratio between two-point and three-point correlation functions [2]. On the other hand, the Wilson coeffi cients have usually been evaluated in continuum perturbation theory. However, we need a cancellation of singularities in the terms $c^{(2)}, A_{4} \propto 1 / a^{2}$, which are again a facet of the renormalon problem. This requires a strictly consistent treatment [3]. Therefore we evaluate the Wilson coeffi cients non-perturbatively as well. This method is particularly suitable for disentangling higher twist contributions. The use of overlap quarks suppresses undesired operator mixings.

In this report we present new numerical results for $c^{(2)}$. For earlier results with Wilson fermions we refer to Ref. [4]. In a previous study with overlap quarks on a $16^{3} \times 32$ lattice [5] some problems persisted, which motivated us to enlarge the lattice to the size $24^{3} \times 48$.

\section{Operator Product Expansion on the lattice}

To be explicit, we apply the Operator Product Expansion (OPE) to a product of electromagnetic currents $J_{\mu}$ between quark states $|\psi(p)\rangle$,

$$
\begin{aligned}
W_{\mu v}(p, q) & =\left\langle\psi(p)\left|J_{\mu}(q) J_{v}^{\dagger}(q)\right| \psi(p)\right\rangle \\
& =\sum_{m} C_{\mu v, i, \mu_{1} \ldots \mu_{n}}^{(m)}(q)\left\langle\psi(p)\left|\mathscr{O}_{i, \mu_{1} \ldots \mu_{n}}^{(m)}\right| \psi(p)\right\rangle .
\end{aligned}
$$

The lower line is a decomposition in terms of local operators $\mathscr{O}^{(m)}$, which characterise the nucleon structure. The index $i=1 \ldots 16$ specifi es the Dirac structure, $\mu_{1} \ldots \mu_{n}$ indicate the momentum components involved, and $m$ distinguishes operators with the same symmetry. Note that the corresponding Wilson coeffi cients $C^{(m)}$ depend solely on the transfer momentum $q$.

A truncation of this expansion at some low operator dimension requires the following scale separation,

$$
p^{2} \ll q^{2} \ll\left(\frac{\pi}{a}\right)^{2}
$$

So far we have been using periodic boundary conditions for the gauge fi eld. Thus a sizable lattice is required to have a set of small momenta $p^{2}$ available. If this scale separation is realised, it justifi es a truncation of the OPE (2.1), which cuts off operators with high derivatives, i.e. high powers of $p$. Here we consider quark bilinears with up to 3 derivatives,

$$
\bar{\psi} \Gamma_{i} \psi, \quad \bar{\psi} \Gamma_{i} D_{\mu_{1}} \psi, \quad \bar{\psi} \Gamma_{i} D_{\mu_{1}} D_{\mu_{2}} \psi, \quad \bar{\psi} \Gamma_{i} D_{\mu_{1}} D_{\mu_{2}} D_{\mu_{3}} \psi
$$


where $\Gamma_{i}$ runs over a basis of the Clifford algebra. This still amounts to an apparently frightening set of $16 \cdot \sum_{d=0}^{3} 4^{d}=1360$ operators. However, we choose the isotropic transfer momenta

$$
\begin{array}{rlrl}
q_{a} & =\frac{\pi}{4 a}(1,1,1,1) & \left(\left|q_{a}\right|\right. & \simeq 4.1 \mathrm{GeV}), \\
q_{b} & =\frac{\pi}{3 a}(1,1,1,1) & \left(\left|q_{b}\right| \simeq 5.5 \mathrm{GeV}\right) .
\end{array}
$$

In this case the symmetry reduces the set of operators to only 67 equivalence classes [5]. We denote them as $C_{1} \ldots C_{67}$, which refer to an ascending number of derivatives:

$$
\begin{aligned}
C_{1} & : \text { no derivative, vanishes in the chiral limit, multiplies } \bar{\psi} \mathbb{\psi} \\
C_{2} \ldots C_{6} & : \text { one derivative, Bjorken scaling } \propto 1 / q^{2} \\
C_{7} \ldots C_{16} & : \text { two derivatives, vanish in the chiral limit } \\
C_{17} \ldots C_{67} & : \text { three derivatives, Bjorken scaling } \propto 1 /\left(q^{2}\right)^{2} .
\end{aligned}
$$

The coeffi cients of terms with an even number of derivatives vanish at quark mass $m=0$ due to chiral symmetry. In the Bjorken limit of large $q^{2}$ the coeffi cients of terms with one (three) derivative(s) are expected to scale as $C_{m} \propto 1 / q^{2}\left(C_{m} \propto 1 /\left(q^{2}\right)^{2}\right)$. Some explicit terms are given in Ref. [5]; for a comprehensive description we refer to Ref. [6].

To separate the scales even better, we have now implemented twisted boundary conditions: thus very small $p^{2}$ become accessible, which enables us to use $q^{2}$ further below the momentum cutoff squared [6]. This allows us to consider for instance $q=\frac{\pi}{6 a}(1,1,1,1)$.

\section{Results for the Wilson coefficients}

In our numerical study we analysed confi gurations that were generated in the quenched approximation with the Lüscher-Weisz gauge action in a volume $V=24^{3} \times 48$, with a physical lattice spacing $a \simeq 0.075 \mathrm{fm}$. To provide fi nite OPE matrix elements, as they occur in eq. (2.1), we fi xed the lattice Landau gauge; this is also favourable to reduce the statistical noise.

For the valence quarks we used overlap fermions with the parameter $\rho=1.4$ (negative mass of the Wilson kernel) at a bare quark mass of 0.028 in lattice units, which corresponds to about $73 \mathrm{MeV}$. The application of chiral lattice fermions suppresses undesired operator mixings, as well as $O(a)$ lattice artifacts.

We denote the number of $p$-sources as $M$, and the number of Wilson coeffi cients to be considered as $C_{1} \ldots C_{N}$, with $N=67$ in our case. So far we evaluated $W_{\mu \nu}$ off-shell for $M=25$ $p$-momentum sources at transfer momentum $q_{a}$ (on different confi gurations), and for $M=10 p$ momentum sources at $q_{b}$. In each case this yields a system constraining the Wilson coeffi cients,

$$
\left(\begin{array}{c}
W^{\left(p_{1}\right)} \\
\cdot \\
\cdot \\
\cdot \\
W^{\left(p_{M}\right)}
\end{array}\right)=\left(\begin{array}{ccc}
\mathscr{O}_{1}^{\left(p_{1}\right)} & \ldots & \mathscr{O}_{N}^{\left(p_{1}\right)} \\
\cdot & \cdots & \cdot \\
\cdot & \cdots & \cdot \\
\cdot & \cdots & \cdot \\
\mathscr{O}_{1}^{\left(p_{M}\right)} & \cdots & \mathscr{O}_{N}^{\left(p_{M}\right)}
\end{array}\right)\left(\begin{array}{c}
C_{1} \\
\cdot \\
\cdot \\
C_{N}
\end{array}\right)
$$

where the elements $W^{\left(p_{i}\right)}$ and $\mathscr{O}_{k}^{\left(p_{i}\right)}$ are $4 \times 4$ matrices. 

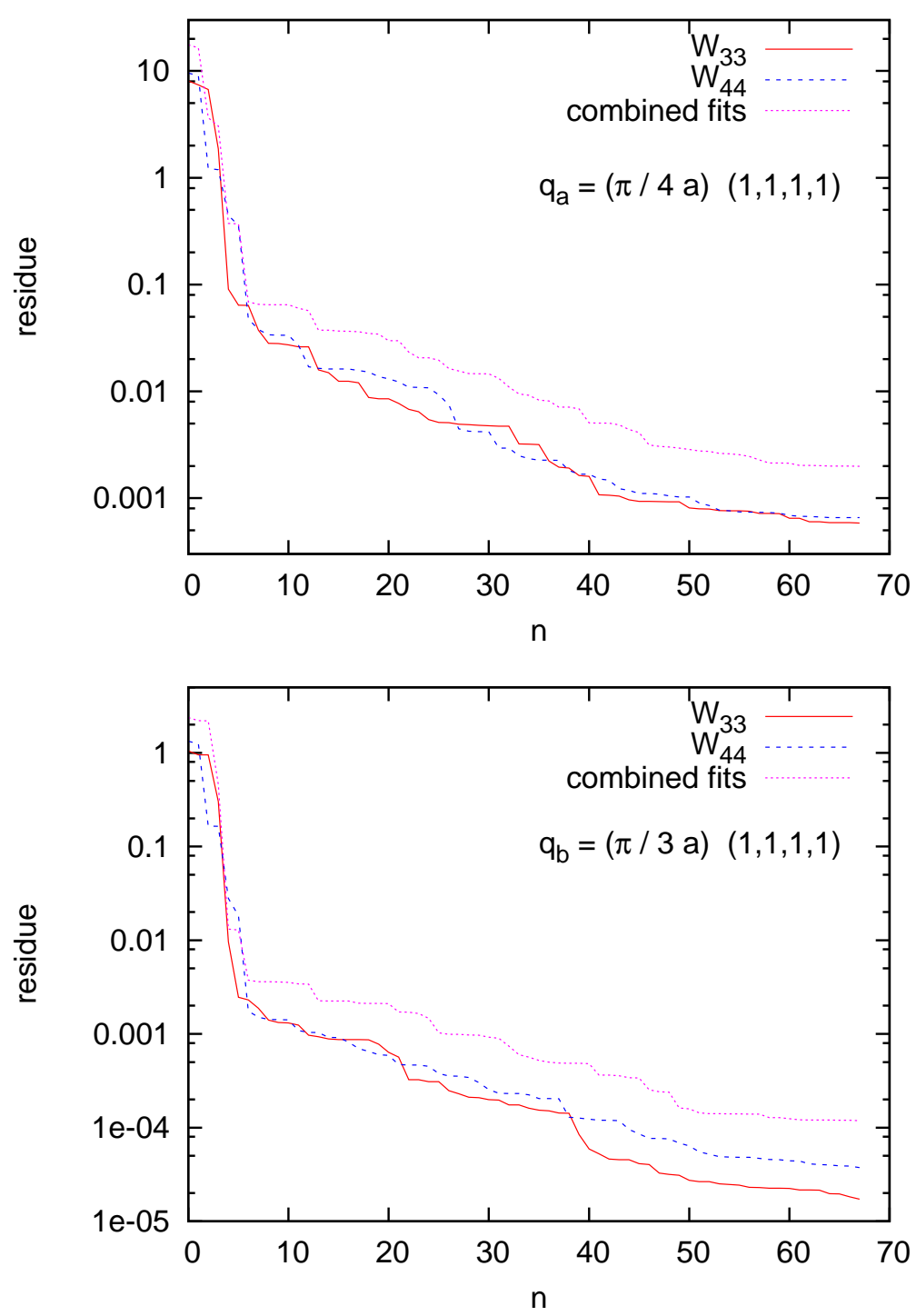

Figure 1: The convergence of the Wilson coefficients as the number of the $n$ most powerful constraints is increased. We show the residues as a function of $n$ for the transfer momentum $q_{a}$ with $25 p$-sources (upper plot), and for $q_{b}$ with $10 p$-sources (lower plot). In both cases we see a convincing convergence before $n$ reaches the deterministic number 67 , which confirms that the extracted Wilson coefficients are trustworthy.

In both cases the system is over-determined since $16 M>N$. We apply Singular Value Decomposition as an established method to analyse such systems [7]. Roughly speaking, this methods selects the $n \leq N$ conditions with the "maximal impact" on the solution $C_{1}, \ldots, C_{N}$. A rapid convergence for increasing $n$ approves a reliable result (i.e. the remaining conditions are negligible). With 12 singular values (analogues of eigenvalues in rectangular matrices [7]) we do observe this feature. As an example Figure 1 shows the convergence in $n$ for the Wilson coeffi cients under consideration, which saturates around $n \approx 50$ for $q_{a}$ and for $q_{b}$. In the latter case, the total number of 
constraints is lower, hence it is easier to satisfy them to a good precision. Therefore the residues ${ }^{1}$ are smaller, but the result is less reliable. Note also that $q_{a}^{2}=\frac{1}{4}(\pi / a)^{2}$ is more promising than $q_{b}^{2}=\frac{4}{9}(\pi / a)^{2}$ in view of the required scale separation (2.2).
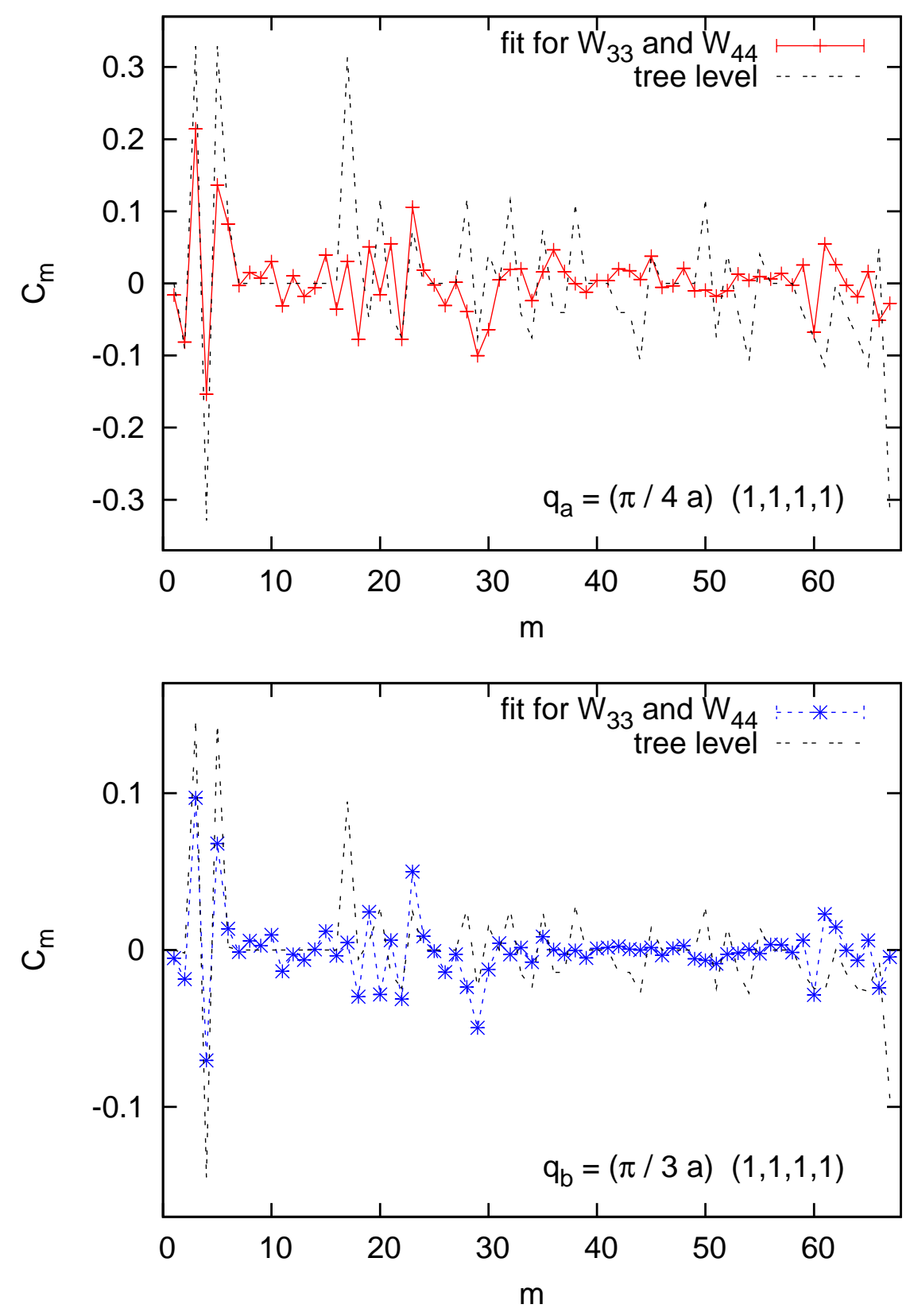

Figure 2: The Wilson coefficients evaluated at momentum transfer $q_{a}$ (upper plot) and $q_{b}$ (lower plot). The results are compared to the corresponding tree level values (with the same overlap Dirac operator). We observe the same pattern, but a significant non-perturbative correction. In the chiral limit $C_{1}$ and $C_{7} \ldots C_{16}$ vanish. The small values that we obtain (with light quarks) for these coefficients provide a consistent picture.

\footnotetext{
${ }^{1}$ The residue is the norm of the difference between the two sides of eq. (3.1) in the given approximation.
} 
There are a number of discrete rotations and reflections which leave our isotropic $q$-momenta (2.4) invariant. For example this allows us to exchange $W_{33}$ with $W_{44}$. As a consequence, specifi c pairs of Wilson coeffi cients belonging to different operators have to coincide; for instance the coeffi cient of $\mathscr{O}_{i, 33}$ in $W_{33}$ is equal to the coeffi cient of $\mathscr{O}_{i, 44}$ in $W_{44}$. We implemented this property in combined fi ts, which have of course somewhat larger residues, see Figure 1.

We now proceed to the actual results for the Wilson coeffi cients obtained from $W_{33}$ and $W_{44}$. The best results emerge from combined fi ts. They are shown in Figure 2, which also displays the corresponding tree level values for comparison.

In the limit of zero quark mass the coeffi cients $C_{1}$ and $C_{7} \ldots C_{16}$ vanish due to chiral symmetry, as we anticipated in Section 2. Since we are dealing with light quarks represented by chiral lattice fermions, we are fairly close to chirality. Therefore it is a stringent consistency test that we do obtain particularly small values for these coeffi cients. That property had not been observed with Wilson fermions [4].

Finally we also test the Bjorken scaling property, which we mentioned in Section 2. We show again the $C_{m}$ obtained at $q_{a}$. We compare them to the Wilson coeffi cients evaluated at $q_{b}$, where $C_{2} \ldots C_{6}$ are enhanced with a factor $q_{b}^{2} / q_{a}^{2}=16 / 9$, and $C_{17} \ldots C_{67}$ are amplifi ed with the square of this factor. The Bjorken re-scaled results are confronted in Figure 3, and we see an impressive similarity, in particular for cases with relatively large $\left|C_{m}\right|$.

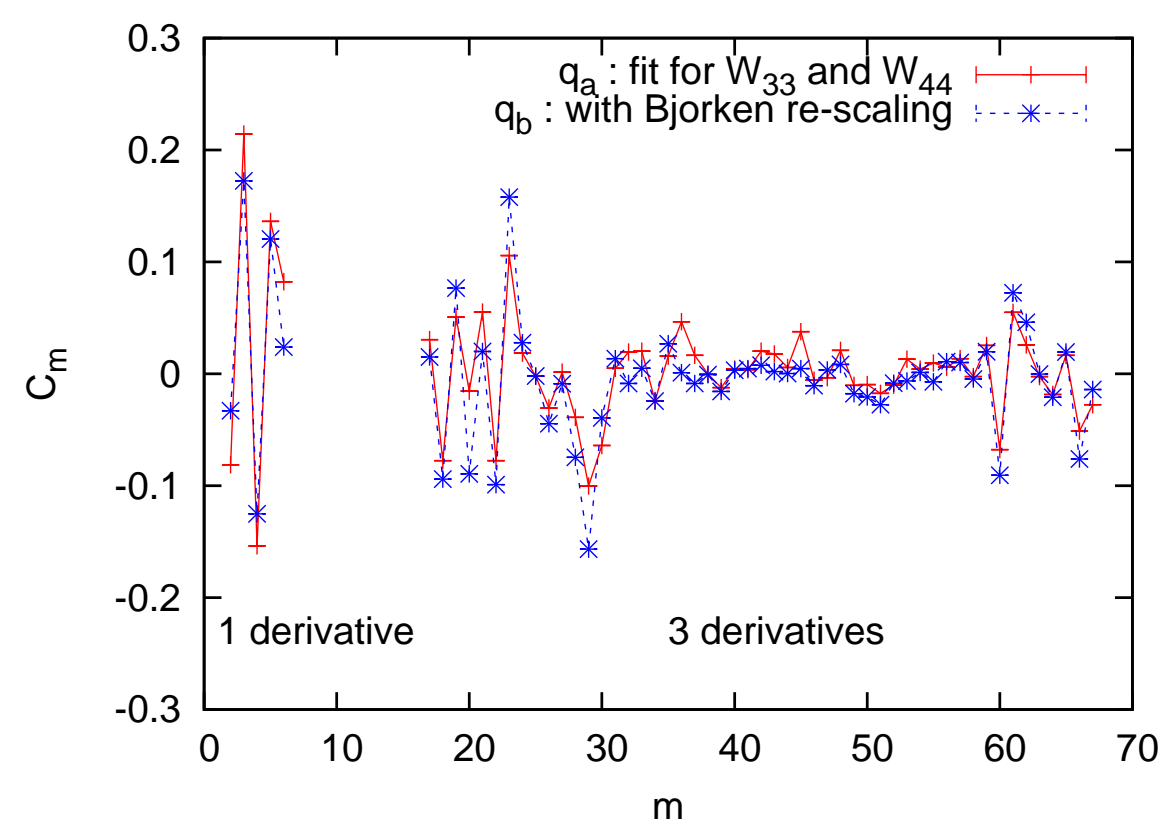

Figure 3: The Wilson coefficients evaluated at momentum transfer $q_{a}$ and $q_{b}$, where in the latter case the coefficients of terms with one or three derivatives are amplified according to the expected scaling in the Bjorken limit. In fact the $C_{m}$ are now in the same range, and we observe a very satisfactory level of agreement. 


\section{Conclusions and outlook}

Our method to evaluate the Wilson coeffi cients non-perturbatively works successfully. We arrived at conclusive non-perturbative results for a considerable set of Wilson coeffi cients. We are continuing to evaluate constraints for additional $q$ and $p$ momenta to further tighten the control over possible artifacts. The use of twisted boundary conditions enables us now to achieve an even better scale separation.

In a fi nal step, the nucleon structure function $\mathscr{M}$ is obtained by Nachtmann integration [8] over $W_{\mu \nu}$. For instance, for the second moment this integral takes the form

$$
\begin{aligned}
\mathscr{M}_{2}(q) & =\frac{3 q^{2}}{(4 \pi)^{2}} \int d \Omega_{q} n_{\mu}\left[W_{\mu v}(q)-\frac{1}{4} \delta_{\mu \nu} W_{\rho \rho}\right] n_{v} \\
& \rightarrow \int_{0}^{1} d x\left[F_{2}\left(x, q^{2}\right)+\frac{1}{6} F_{L}\left(x, q^{2}\right)\right],
\end{aligned}
$$

where the Bjorken limit is taken in the lower line. The projection vector has length $n^{2}=1$. For a different projection one obtains $\int_{0}^{1} d x\left[F_{2}-\frac{3}{2} F_{L}\right]$ instead. Thus the combination of different projections determines the longitudinal structure function $F_{L}=F_{2}-2 x F_{1}$.

With completed data, we will obtain a fully non-perturbative and consistent evaluation of the Nucleon Structure Functions [6].

Acknowledgement : The computations were performed on the clusters of the "Norddeutscher Verbund für Hoch- und Höchstleistungsrechnen" (HLRN). We thank Hinnerk Stüben for technical assistance.

\section{References}

[1] A.I. Vainshtein and V.I. Zakharov, Phys. Rev. Lett. 73 (1994) 1207 (Erratum ibid. 75 (1995) 3588). E. Stein, M. Maul, L. Mankiewicz and A. Schäfer, Nucl. Phys. B 536 (1998) 318.

V.M. Braun, E. Gardi and S. Gottwald, Nucl. Phys. B 685 (2004) 171.

[2] M. Göckeler, R. Horsley, D. Pleiter, P.E.L. Rakow and G. Schierholz, Phys. Rev. D 71 (2005) 114511.

[3] G. Martinelli and C.T. Sachrajda, Nucl. Phys. B 478 (1996) 660.

[4] S. Capitani, M. Göckeler, R. Horsley, H. Oelrich, D. Petters, P.E.L. Rakow and G. Schierholz, Nucl. Phys. (Proc. Suppl.) 73 (1999) 288. D. Petters, Ph.D. Thesis, Freie Universität Berlin (2000). M. Göckeler, R. Horsley, H. Perlt, P.E.L. Rakow, G. Schierholz and A. Schiller (QCDSF Collaboration) PoS(LAT2006)119.

[5] W. Bietenholz, N. Cundy, M. Göckeler, R. Horsley, H. Perlt, D. Pleiter, P.E.L. Rakow, C.J. Roberts, G. Schierholz, A. Schiller and J.M. Zanotti (QCDSF Collaboration), PoS(LAT2007)159.

[6] QCDSF Collaboration, in preparation.

[7] W.H. Press, S.A. Teukolsky, W.T. Vetterling and B.P. Flannery, 'Numerical Recipes”, Cambridge University Press, Cambridge UK (1989).

[8] O. Nachtmann, Nucl. Phys. B 63 (1973) 273. 